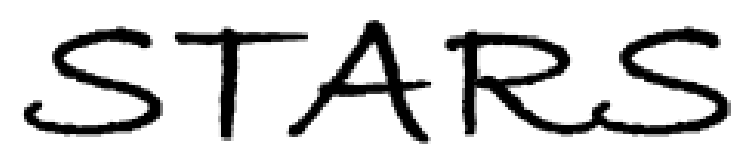

University of Central Florida

STARS

2013

\title{
An Event-related Potential Investigation Of Error Monitoring In Adults With A History Of Psychosis
}

Chi Chan

University of Central Florida

Part of the Clinical Psychology Commons

Find similar works at: https://stars.library.ucf.edu/etd

University of Central Florida Libraries http://library.ucf.edu

This Masters Thesis (Open Access) is brought to you for free and open access by STARS. It has been accepted for inclusion in Electronic Theses and Dissertations, 2004-2019 by an authorized administrator of STARS. For more information, please contact STARS@ucf.edu.

\section{STARS Citation}

Chan, Chi, "An Event-related Potential Investigation Of Error Monitoring In Adults With A History Of Psychosis" (2013). Electronic Theses and Dissertations, 2004-2019. 2827.

https://stars.library.ucf.edu/etd/2827

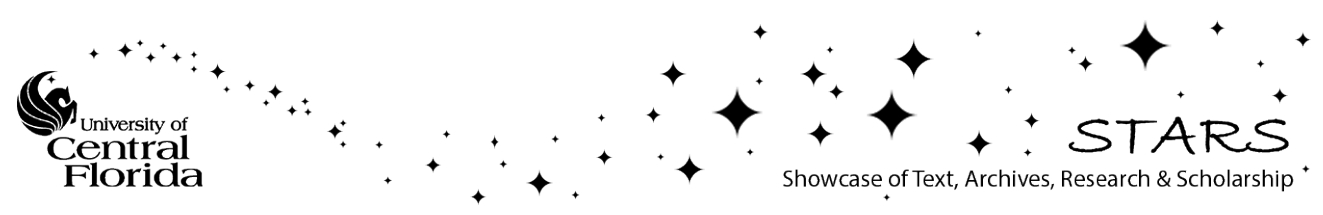




\title{
AN EVENT-RELATED POTENTIAL INVESTIGATION OF ERROR MONITORING IN ADULTS WITH A HISTORY OF PSYCHOSIS
}

\author{
by \\ CHI CHAN \\ B.A. Temple University, 2008
A thesis submitted in partial fulfillment of the requirements for the degree of Master of Science in the Department of Psychology in the College of Sciences at the University of Central Florida Orlando, Florida

Fall Term 2013 


\begin{abstract}
Metacognition, which involves monitoring and controlling of one's thoughts and actions, is essential for guiding behavior and organization of information. Deficits in self-monitoring have been suggested to lead to psychosis and poor functional outcome. Abnormalities in eventrelated potentials originating from the anterior cingulate cortex (ACC), a brain region associated with error detection, have been consistently reported in individuals with schizophrenia during error monitoring tasks. This study sought to examine whether these abnormalities are present in individuals with a history of psychosis across diagnostic categories and whether they are associated with subjective appraisal of self-performance and personality traits related to psychosis. The error-related negativity (ERN), the correct response negativity (CRN), and the error positivity (Pe) were recorded in 15 individuals with a history of psychosis (PSY) and 12 individuals without a history of psychosis (CTR) during performance on a flanker task.

Participants also continuously rated their performance on the task and completed the Schizotypal Personality Questionnaire - Brief Revised (SPQ-BR). Compared with the CTR group, the PSY group exhibited reduced ERN and Pe amplitudes during error trials, but normal CRN and Pe amplitudes during correct trials. The PSY group also was less accurate at identifying their errors than the CTR group but just as accurate at identifying correct responses. Across all participants, smaller ERN amplitudes were associated with greater scores on the Disorganized factor of the SPQ-BR and smaller Pe amplitudes were associated with greater scores on the Cognitive Perceptual factor of the SPQ-BR. Individuals with a history of psychosis regardless of diagnosis demonstrated abnormal neural activity during error monitoring. Error monitoring deficits may be associated with vulnerability for psychosis across disorders.
\end{abstract}




\section{TABLE OF CONTENTS}

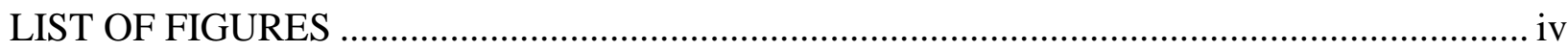

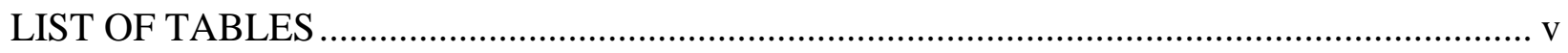

CHAPTER 1: INTRODUCTION ................................................................................. 1

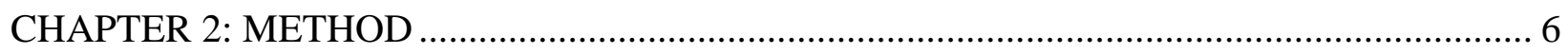

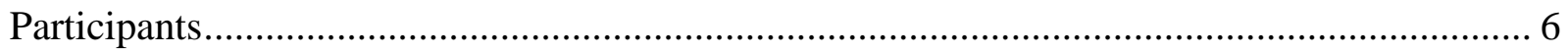

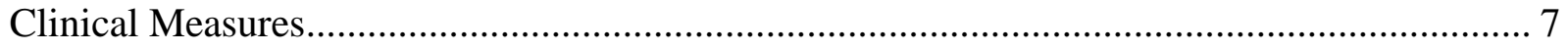

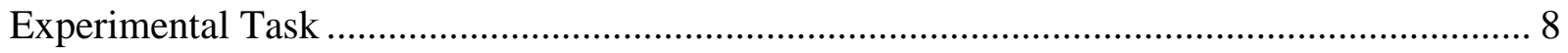

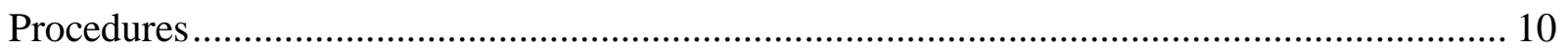

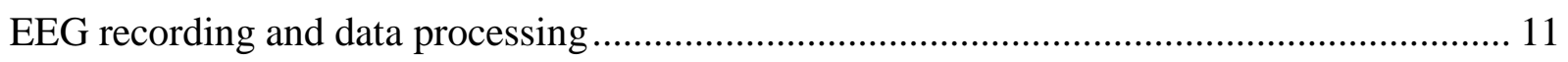

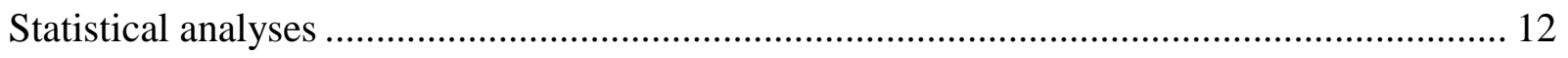

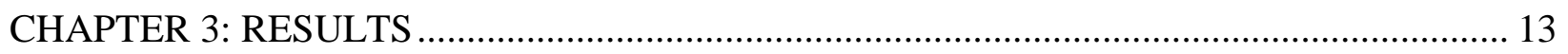

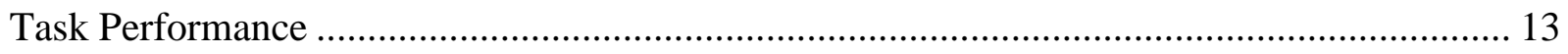

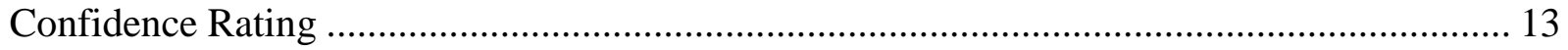

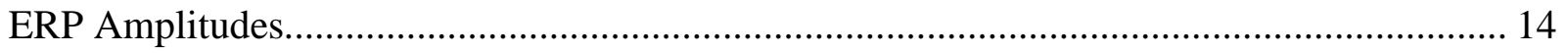

ERP Amplitude Correlation with Schizotypy ........................................................... 16

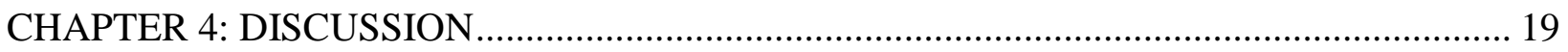

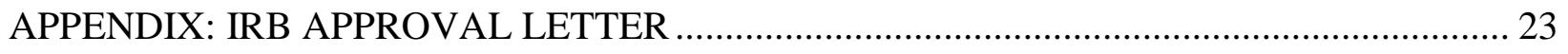

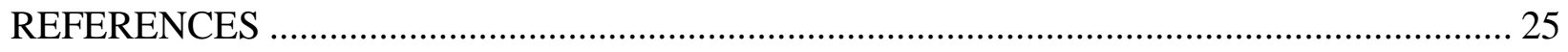




\section{LIST OF FIGURES}

Figure 1: Sample of an incongruent trial. ...................................................................... 9

Figure 2: Mean accuracy of confidence ratings for error and correct trials............................ 14

Figure 3: Grand average waveforms at the FCz site........................................................ 15

Figure 4: Mean amplitude of the Pe at site $\mathrm{Cz}$ during error and correct trials.......................... 16

Figure 5: Correlation between the SPQ-BR Disorganized factor and ERN amplitude............... 17

Figure 6: Correlation between the SPQ-BR Cognitive Perceptual Factor and Pe amplitude....... 18 


\section{LIST OF TABLES}

Table 1 Participant Demographic Data.......................................................................... 7 


\section{CHAPTER 1: INTRODUCTION}

Metacognition refers to "our knowledge about how we perceive, remember, think, and act - that is, what we know about what we know" (Metcalfe \& Shimamura, 1994). It is conceptualized as involving two important processes: monitoring and control. Monitoring refers to the evaluation of one's own cognitive functioning and control refers to the way in which behavior is guided by self-evaluation (Nelson \& Narens, 1990). These processes are essential for guiding goal-directed action and organization of internal and external information. In schizophrenia, failed or inadequate monitoring (or misattribution) of self-action and thought has been related to positive symptomology (e.g., Frith, 1987; Frith \& Done, 1989). Recently, researchers have argued that metacognition is an important factor both mediating and moderating associations between neurocognitive deficits and functional outcome in schizophrenia (Green, Kern, Braff, \& Mintz, 2000; Koren, Seidman, Goldsmith, \& Harvey, 2006; Lysaker et al., 2010; Lysaker et al., 2013; Penn, Corrigan, Bentall, Racenstein, \& Newman, 1997).

Error monitoring is particularly important, as recognizing a mistake and reacting appropriately is critical for adequate functioning. Studies on individuals with schizophrenia demonstrate less error monitoring effort (Silver et al., 2006; Silver \& Goodman, 2007), less accuracy in discriminating between real or imagined self-actions (Gawęda, Woodward, Moritz, \& Kokoszka, 2013), less accurate corrective action during task completion (Hommes et al., 2011), and imprecise self-report of confidence in responses, as characterized by overconfidence in errors and reduced confidence in correct responses (Kircher, Koch, Stottmeister, \& Durst, 2007; Moritz, Woodward, \& Ruff, 2002; Moritz \& Woodward, 2006a).

Attempts to understand the underlying neural mechanism of metacognition using event- 
related potentials (ERP; Dehaene, Posner, \& Tucker, 1994) and other psychophysiological techniques (Carter et al., 1998; Holroyd et al., 2004, Miltner et al., 2003) have found unique brain reactions originating from the anterior cingulate cortex (ACC) during tasks that require error monitoring. These tasks often produce "“action slips' - that is, fast, impulsive errors, based on insufficient processing of the relevant stimuli" (van Veen \& Carter, 2006). In this type of task, the error-related negativity $(\mathrm{ERN})$ is the initial negative deflection in the ERP following an error (Gehring, Goss, Coles, Meyer, \& Donchin, 1993), peaks approximately 50-150 ms following a response, and has a maximal inflection at frontocentral electrode sites (e.g., Bernstein, Scheffers, \& Coles, 1995; Falkenstein, Hoormann, Christ, \& Hohnsbein, 2000; Gehring et al., 1993; Morris, Yee, \& Nuechterlein, 2006; Nieuwenhuis, Ridderinkhof, Blom, Band, \& Kok, 2001). It is more prominent when accuracy is emphasized over speed (Falkenstein et al., 2000; Gehring et al., 1993; Morris et al., 2006). The presence of the ERN in different paradigms supports the idea of a general error-processing system that acts regardless of response modality. The ERN is generally associated with the subconscious awareness of error (Nieuwenhuis et al., 2001), although some studies have also found it to be associated with subjective appraisal of accuracy (Scheffers \& Coles, 2000) and post-error slowing, a decrease in reaction time (RT) following errors that is thought to be related to remedial action (Debener et al., 2005; Gehring et al., 1993).

Sometimes a negative ERP component also is seen following correct responses, and thus referred to as the correct response negativity $(\mathrm{CRN})$. The $\mathrm{CRN}$ shares the same time course and electrode sites as the ERN (Coles, Scheffers, \& Holroyd, 2001; Falkenstein et al., 2000; Vidal, Hasbroucq, Grapperon, \& Bonnet, 2000) and its presence suggests that there may be some type 
of error-monitoring activity even if an error was not actually executed. It has been suggested as a result of detection of partial errors (e.g., when the idea of an incorrect response occurred but the correct response was actually executed), or as an artifact of measurement - when stimulusrelated activity continues into the response-locked segments used to measure the ERN (see Coles et al., 2001). If the former is the case, in which an incorrect response may have begun (either cognitively or physically) but the actual response given is correct, then the CRN may represent a successful metacognitive process encompassing both monitoring and control. The monitoring process detects a mismatch between the correct response and the beginning of an incorrect response, and the control process takes over to successfully correct this mismatch.

Following the initial negativity, a positively deflected ERP peaking at approximately $160-500 \mathrm{~ms}$ after a response is referred to as the error positivity (Pe). It is maximal at parietocentral electrode sites and more pronounced following error responses (Falkenstein, Hohnsbein, Hoormann, \& Blanke, 1991; Falkenstein et al., 2000; Vidal et al., 2000). In contrast to the ERN, the Pe is generally associated with the conscious detection of errors (Nieuwenhuis et al., 2001) and the remedial action of post-error slowing (Hajcak, McDonald, \& Simons, 2003; Nieuwenhuis et al., 2001).

In patients with schizophrenia, a reduced ERN amplitude has been demonstrated using a wide variety of cognitive tasks, including flanker tasks (Horan, Foti, Hajcak, Wynn, \& Green, 2011; Kopp \& Rist, 1999; Morris et al., 2006), a Stroop task (Alain, McNeely, He, Christensen, \& West, 2002), and the go/no-go paradigm (Bates, Kiehl, Laurens, \& Liddle, 2002; Mathalon, Jorgensen, Roach, \& Ford, 2009). Moreover, the CRN amplitude also appears to be abnormal in schizophrenia, which has been reported as either increased (Alain et al., 2002; Mathalon et al., 
2002; Morris et al., 2006) or decreased relative to controls (Bates et al., 2002). The reduction in ERN amplitude in schizophrenia samples appears not to be related to overall accuracy on the task, as it is present regardless of whether accuracy is comparable between patients and controls. It is also not a result of a general reduction in brain activity, evidenced by studies that have found increased CRN amplitude coinciding with reduced ERN amplitude in the same schizophrenia samples (Mathalon et al., 2002; Morris et al., 2006). Pe amplitude appears to be normal across schizophrenia samples and tasks (Alain et al., 2002; Mathalon et al., 2002; Morris et al., 2006), while post-error reaction time slowing has been found in some studies (Alain et al., 2002; Kerns et al., 2005), but not all (Bates et al., 2002; Mathalon et al., 2002; Morris et al., 2006).

Psychosis is a necessary symptom for a diagnosis of schizophrenia according to the DSM-5; however, it is not unique to schizophrenia as it can also occur in mood and other disorders. Given the findings in schizophrenia samples, abnormal brain responses during error monitoring may be a vulnerability marker for psychosis. The Research Domain Criteria (RDoC) initiative advanced by the National Institute of Mental Health seeks to understand psychopathology, presumably resulting from common neural circuit dysfunction, across rather than within disorders. The purpose of the present study is to use a variation of the flanker task (Eriksen \& Eriksen, 1974; see method section below) to examine error monitoring in individuals with and without a history of psychosis using behavioral and psychophysical approaches. Based on evidence from the existing literature, we hypothesize that: (1) similar to the imprecise confidence in response found in schizophrenia, the psychosis group (PSY) will be less accurate in identifying their errors than the comparison control group (CTR); (2) PSY will exhibit reduced ERN amplitude, increased CRN amplitude, but normal Pe amplitude; and (3) given that error 
monitoring deficits have been suggested to lead to psychosis, we predict that abnormalities in ERP amplitudes will be related to personality factors that are associated with psychosis across the entire sample. Specifically, reduced ERN and increased CRN will be related to personality factors associated with psychosis whereas Pe amplitude will be unrelated. 


\title{
CHAPTER 2: METHOD
}

\author{
Participants
}

Participants were recruited from the community and local mental health facilities via local newspaper advertisements, referrals, flyers, and online advertisements. Potential participants were screened over the telephone and excluded if they were not native English speakers or reported a history of medical conditions or traumatic head injury that may affect brain functioning or their ability to perform the task. Comparison controls were also excluded if they reported that they or any of their biological relatives had ever been prescribed an antipsychotic medication or if they reported that a biological family member had ever received a diagnosis of schizophrenia, schizoaffective disorder, or bipolar disorder.

A total of 22 individuals with a history of psychosis and 33 individuals without a history of psychosis completed the study. Data from 7 individuals with a history of psychosis were excluded for having fewer than 5 errors (see Foti, Kotov, \& Hajcak, 2013) and data from 21 comparison controls were excluded for not completing the task $(n=2)$, equipment problems $(n=$ 1), and having fewer than 5 error trials $(\mathrm{n}=18)$. The final sample consisted of 15 individuals with a history of psychosis and 12 individuals without a history of psychosis. Groups did not differ significantly on gender, age, ethnicity, education, or parental education (see Table 1). Groups also did not differ significantly on the number of epochs in the ERP average for error and correct trials at each electrode site (all $p$ 's $>.05$ ).

Primary diagnoses in the PSY group included bipolar I disorder with a history of psychosis $(\mathrm{n}=5)$, delusional disorder $(\mathrm{n}=1)$, schizophrenia $(\mathrm{n}=3)$, and schizoaffective disorder $(\mathrm{n}=6)$. Primary diagnoses in the CTR group included bipolar I disorder without a history of 
psychosis $(n=3)$, bipolar II disorder $(n=1)$, generalized anxiety disorder $(n=1)$, social phobia $(n=1)$, major depressive disorder $(n=1)$, and no diagnosis $(n=5)$. Individuals in the PSY group were treated with atypical antipsychotic medication $(n=11)$, a combination of typical and atypical antipsychotic medications $(n=3)$, and no antipsychotic medication $(n=1)$. One individual in the CTR group was prescribed an atypical antipsychotic medication, although a history of psychosis was denied. No other individual in the CTR group was prescribed an antipsychotic medication.

Table 1

Participant Demographic Data

\begin{tabular}{lccc}
\hline & PSY & CTR & \\
& $(\mathrm{n}=15)$ & $(\mathrm{n}=12)$ & Statistics \\
\hline Gender (\% Female) & $66.7 \%$ & $66.7 \%$ & $X^{2}(1)=.000, p=1.0$ \\
\hline Age, $M(S D)$ & $36.0(8.2)$ & $37.2(8.8)$ & $t(25)=.356, p=.725$ \\
\hline Ethnicity & & & \\
\% Caucasian & $53.3 \%$ & $75.0 \%$ & $X^{2}(2)=1.410, p=.494$ \\
\% African American & $26.7 \%$ & $16.7 \%$ & \\
\% Mixed & $20.0 \%$ & $8.3 \%$ & \\
\hline Education (years), $M(S D)$ & $12.8(3.4)$ & $14.5(1.9)$ & $t(25)=1.54, p=.136$ \\
\hline Parental education (years), $M(S D)$ & $13.3(2.3)$ & $13.7(2.0)$ & $t(24)=.491, p=.628$ \\
\hline
\end{tabular}

\section{Clinical Measures}

The Structured Clinical Interview for DSM-IV Axis I and the Avoidant, Paranoid, and Schizotypal Personality Disorder sections of the Structured Clinical Interview for DSM-IV Axis II Disorders (SCID-I and SCID-II; First, Gibbon, Spitzer, Williams, \& Benjamin, 1997; First, Spizter, Gibbon, Williams, \& Janet, 1996) were used to arrive at a consensus diagnosis by three 
investigators (C.C., B.T., and J.B.) during weekly case conferences. The Schizotypal Personality Questionnaire-Brief Revised (SPQ-BR; Cohen, Matthews, Najolia, \& Brown, 2010), a 32-item self-report questionnaire derived from the original SPQ (Raine, 1991), was used to assess traits found in schizotypal personality disorder consistent with the DSM-IV diagnostic criteria. Seven subscales developed from factor analysis load onto three superordinate factors including the Cognitive-Perceptual, Disorganized, and Interpersonal factors. The SPQ-BR was favored over other symptom severity measures (e.g., Brief Psychiatric Rating Scale) because whereas other measures assess recent psychopathology, the SPQ-BR assesses personality traits associated with psychosis that can be represented on a continuum and may be sensitive to psychosis-proneness in individuals without a history of a clinical level of psychotic symptoms.

\section{Experimental Task}

E-Prime 2.0 Professional software (Psychology Software Tools, Sharpsburg, PA) was used to present a modified version of the flanker task (Eriksen \& Eriksen, 1974), which requires participants to respond to a particular stimulus (the target) while ignoring other irrelevant but distracting stimuli (the flankers). The task was modified to maximize relevant ERP amplitudes and to elicit a sufficient number of errors for analysis. Both accuracy and speed were emphasized by instructing participants to "respond as quickly and accurately as possible" and the difficulty of the task was increased by setting a relatively short time limit, presenting the flankers prior to the target, decreasing spatial distance between the target and the flankers, and increasing the number of flankers. Each trial began with a $1 \mathrm{~cm}$ fixation cross displayed in the center of the screen for $1000 \mathrm{~ms}$. The flankers, which were four horizontally arranged equilateral triangles (two on each side of center target) with sides of $21 \mathrm{~mm}$ appeared for $100 \mathrm{~ms}$, followed by the entire array of 
flankers and the target (which is the same size and shape as the flankers), appearing together for $50 \mathrm{~ms}$. The flankers were either pointed in the same direction as the target (congruent condition) or in the opposite direction (incongruent condition). The distance between each triangle was 21 mm. Participants were allowed $1000 \mathrm{~ms}$ to respond. See Figure 1 for a visual depiction of the task and stimuli.

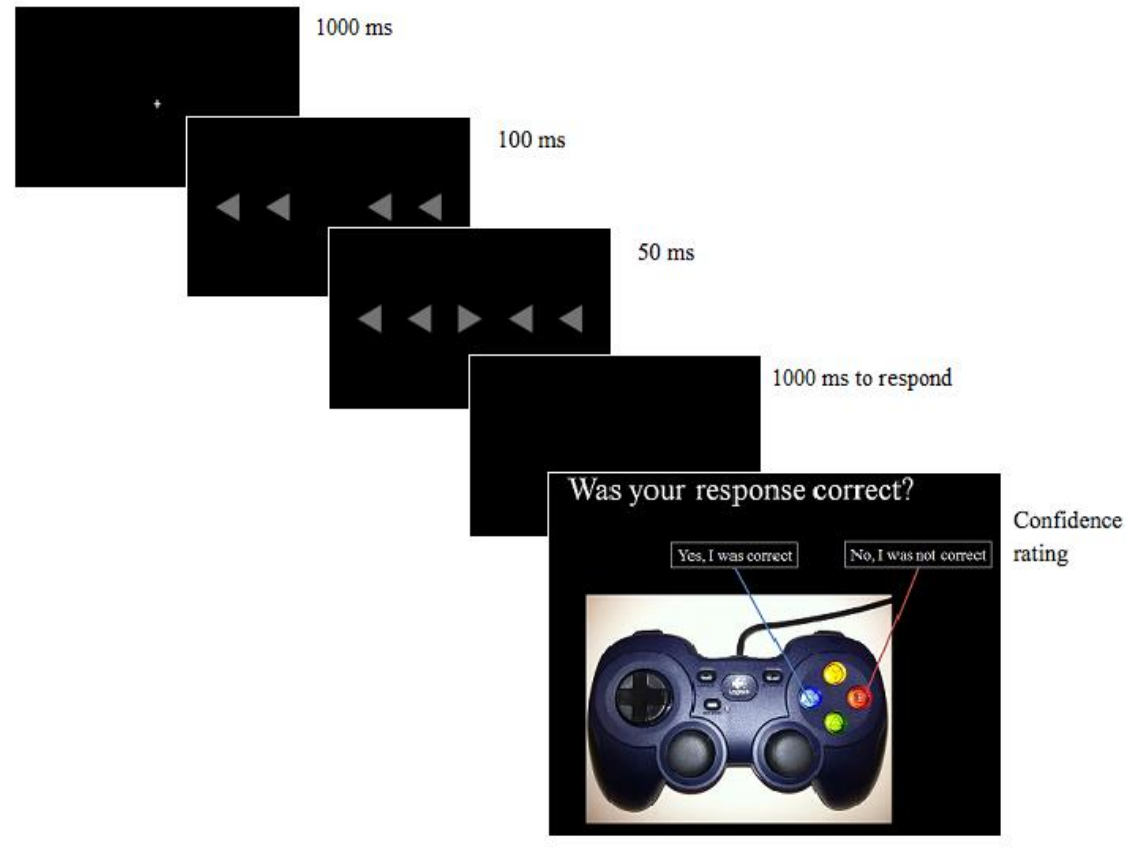

Figure 1: Sample of an incongruent trial.

A videogame controller served as the response device. Participants were instructed to press the left shoulder button (with left index finger) if the target triangle is pointing to the left and the right shoulder button (with right index finger) if it is pointing to the right. If the participant responded within the time limit, they were asked to rate their confidence in their response by answering the question "Was your response correct?" They responded by pressing 
buttons corresponding to the statement "Yes, my response was correct" or "No, my response was not correct." If the participant did not respond within the time limit, a message instructing them to "Respond as quickly and accurately as possible" appeared for $2000 \mathrm{~ms}$. The inter-trial interval following either of these messages was randomly selected from a range of 1000 to $2000 \mathrm{~ms}$. The four different types of trials (congruent or incongruent, target pointing to the left or to the right) were presented in random order with equal probability. All flanker and target stimuli were gray presented against a black background.

\section{Procedures}

This study was conducted in two in-person sessions. During the first session, informed consent, demographic information, and clinical characteristics were obtained. Visual acuity was assessed using the Snellen visual acuity wall chart and participants were excluded if they did not have at least 20/40 vision. The participant completed the experimental task during the second session.

The experimental task was performed in a semi-dark, interior room with no windows. EEG and electrooculogram (EOG) recordings were obtained from a 32-channel electrode cap (Neuroscan Synamps2, Charlotte, NC). Electrode gel and abrading procedures were applied to achieve less than $5 \mathrm{kOhms}$ of impedance in each electrode. Participants were seated $1 \mathrm{~m}$ from a 26" widescreen LCD monitor running at a $75 \mathrm{~Hz}$ refresh rate. Instructions were written on the computer screen as well as read to the participants and included visual aids. Participants completed 16 practice trials to ensure understanding of the task. The experimental task consisted of 4 blocks of 60 trials each, totaling 240 trials. There were self-timed breaks between blocks and the experimental task lasted approximately 30 minutes. 
EEG recording and data processing

EEG and electrooculogram (EOG) were continuously recorded with the Neuroscan Synamps2 (Charlotte, NC) and Neuroscan 4.5 software. EEG and EOG activities were acquired using DC recording at a rate of $1,000 \mathrm{~Hz}$ with a low pass filter of $100 \mathrm{~Hz}$ and no high pass filter. Data were actively referenced to the bilateral mastoid processes. Response-locked epochs beginning at $-200 \mathrm{~ms}$ before response onset and continuing to $700 \mathrm{~ms}$ post-response were created for each trial. Scalp electrodes were processed with a high pass filter of $1 \mathrm{~Hz}$ and a low pass filter of $10 \mathrm{~Hz}$, while EOG electrodes was processed with a high pass filter of $0.05 \mathrm{~Hz}$ and a low pass filter of $30 \mathrm{~Hz}$. A notch filter of $60 \mathrm{~Hz}$ was applied to all electrodes. Single-trials were inspected and rejected for eye blinks and large eye movements using a semi-automatic procedure. Epochs contaminated by other artifacts were rejected, including those that contained more than a $120 \mu \mathrm{V}$ max/min difference within $200 \mathrm{~ms}$ or less than $0.5 \mu \mathrm{V}$ within $100 \mathrm{~ms}$. Baseline correction was conducted using a -150 to $-50 \mathrm{~ms}$ pre-response window.

For each participant, averages were computed for the correct and incorrect responses separately. Based on existing literature, amplitudes of the ERN and CRN were measured from frontocentral electrode sites $\mathrm{Fz}$ and $\mathrm{FCz}$, and $\mathrm{Pe}$ was measured from parietocentral electrode sites $\mathrm{Cz}$ and Pz. The ERN and the CRN mean amplitudes were measured as the average of a $50 \mathrm{~ms}$ window centered on the largest negative peak in the period from $0 \mathrm{~ms}$ to $150 \mathrm{~ms}$ post-response. The Pe was measured as the average of a $50 \mathrm{~ms}$ window centered on the largest positive peak in the period from $170 \mathrm{~ms}$ to $400 \mathrm{~ms}$ post-response. 


\section{Statistical analyses}

SPSS Version 20 was used for all data analysis. Participants were excluded from data analysis for poor performance ( $<75 \%$ accuracy) and for having less than the minimum 5 error trials to establish fair reliability of the ERN (see Foti et al., 2013). First, planned comparisons were conducted based on our hypotheses. Next, mixed ANOVAs were conducted to examine the modulation of confidence rating accuracy, ERP amplitudes, and site across error and correct trials, and the differential effects of these factors in individuals with and without a history of psychosis. The between-subjects factor was group (PSY or CTR) and the within-subjects factors were accuracy of confidence ratings (during correct trials or incorrect trials), trial accuracy (correct or incorrect responses), and site (Fz or FCz for the ERN/CRN and Pz, or $\mathrm{Cz}$ for the Pe). Examination of reaction times excluded trials following no response trials, as the message "Respond as quickly and accurately as possible" may confound changes in RT due to the message with changes in RT due to the participant's evaluation of the previous trial. Pearson's product-moment correlations were conducted to assess the associations between ERN, CRN, and Pe amplitudes and each of the three factor scores of the SPQ-BR. Two-tailed tests and an alpha level of .05 were used for all analyses. 


\section{CHAPTER 3: RESULTS}

The assumption of normality was tested for each variable by examining skewness, kurtosis, and outliers. The Shapiro-Wilk test for normality suggested reasonable normality for all variables of interest (all $p s>.07)$ and there were no significant outliers as defined by values that fell \pm 3 standard deviations from the mean.

\section{Task Performance}

Consistent with the existing literature, the PSY group $(M=91.15, S D=6.55)$ did not differ from the CTR $(M=91.38, S D=6.18)$ group on the percentage of correct responses, $F(1$, $25)=.008, p=.929, \dot{\eta} p^{2}<.001$. Across groups, error rates were higher in incongruent trials, $F(1$, $25)=36.53, p<.001, \dot{\eta} p^{2}=.59$, reaction time was faster on error trials, $F(1,25)=25.23, p<$ $.001, \dot{\eta} p^{2}=.50$, and post-error slowing was demonstrated, $F(1,25)=14.42, p=.001, \dot{\eta} p^{2}=.37$. None of these effects interacted with group (all $p$ 's $>.19$ ).

\section{Confidence Rating}

A 2 (Group) X 2 (Accuracy of confidence ratings) mixed Analysis of Variance (ANOVA) revealed a significant interaction between group and accuracy of confidence ratings, $F(1,25)=4.40, p=.046, \dot{\eta} p^{2}=.150$. Comparisons of the accuracy of confidence ratings during error and correct trials separately in the two groups indicated that the PSY group was significantly less accurate on their confidence ratings than the CTR group during error trials, $F(1$, $25)=4.93, p=.036, \dot{\eta} p^{2}=.165$, but not during correct trials, $p=.232, \dot{\eta} p^{2}=.057$ (see Figure 2). 


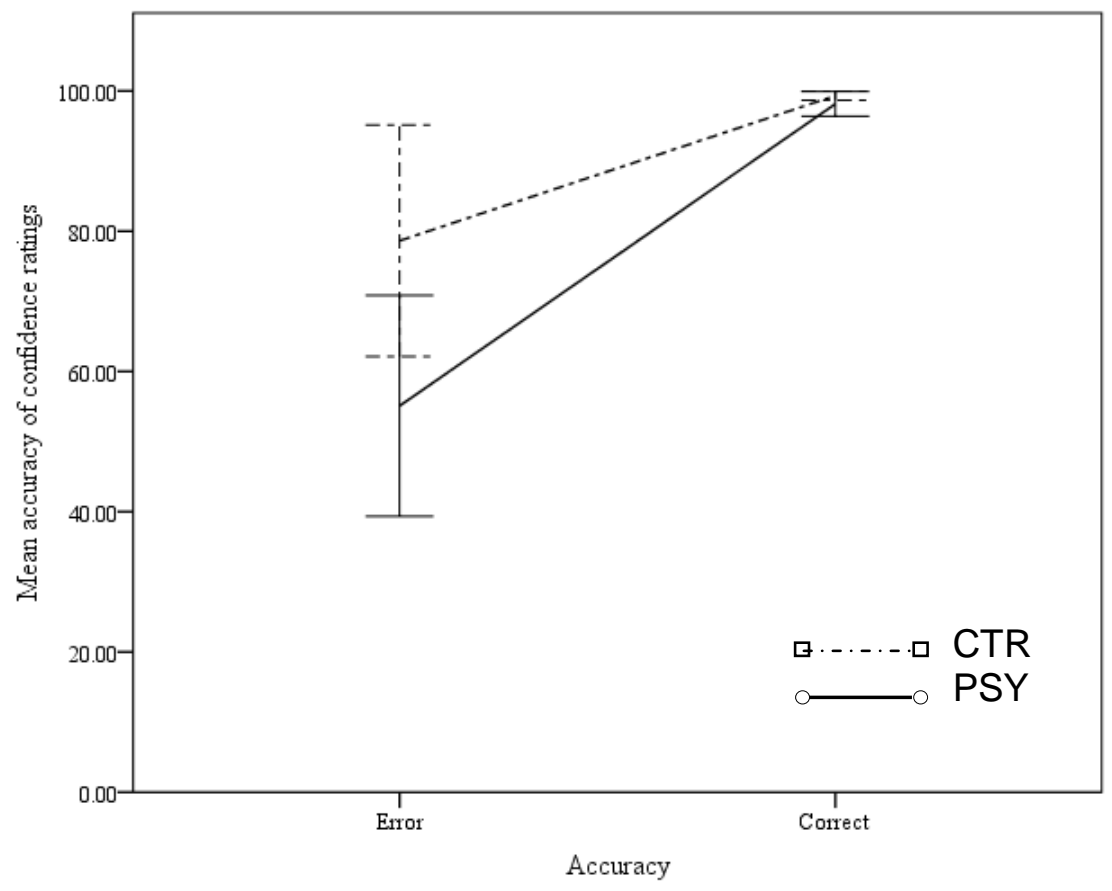

Figure 2: Mean accuracy of confidence ratings for error and correct trials. PSY = individuals with a history of psychosis; CTR = comparison controls. Error bars represent $95 \%$ confidence interval.

\section{ERP Amplitudes}

One participant in the PSY group had fewer than 5 error trials for averaging the ERN at electrode site Fz due to artifact rejection; therefore, that participant's data were excluded for analyses involving the ERN at Fz but included for all other analyses.

Grand average waveforms are presented in Figure 3. Planned comparison $t$-tests revealed that the ERN amplitude was significantly reduced in PSY at FCz, $t(25)=2.31, p=.029, d=.90$ and approached significance at Fz, $t(24)=1.79, p=.085, d=.70$ (see Figure 2). In contrast, CRN amplitudes were not significantly different at either $\mathrm{FCz}, t(25)=.497, p=.623, d=.19$ or $\mathrm{Fz}, t(25)=.749, p=.461, d=.29 . \mathrm{A} 2$ (Group) X 2 (Accuracy of trials) X 2 (Site) mixed 
ANOVA revealed no significant two or three-way interactions (all $p>.13$ ). There was a main effect of accuracy, $F(1,24)=7.52, p=.011, \dot{\eta} p^{2}=.239$, indicating that the ERN is more prominent than the CRN across participants and electrode sites.

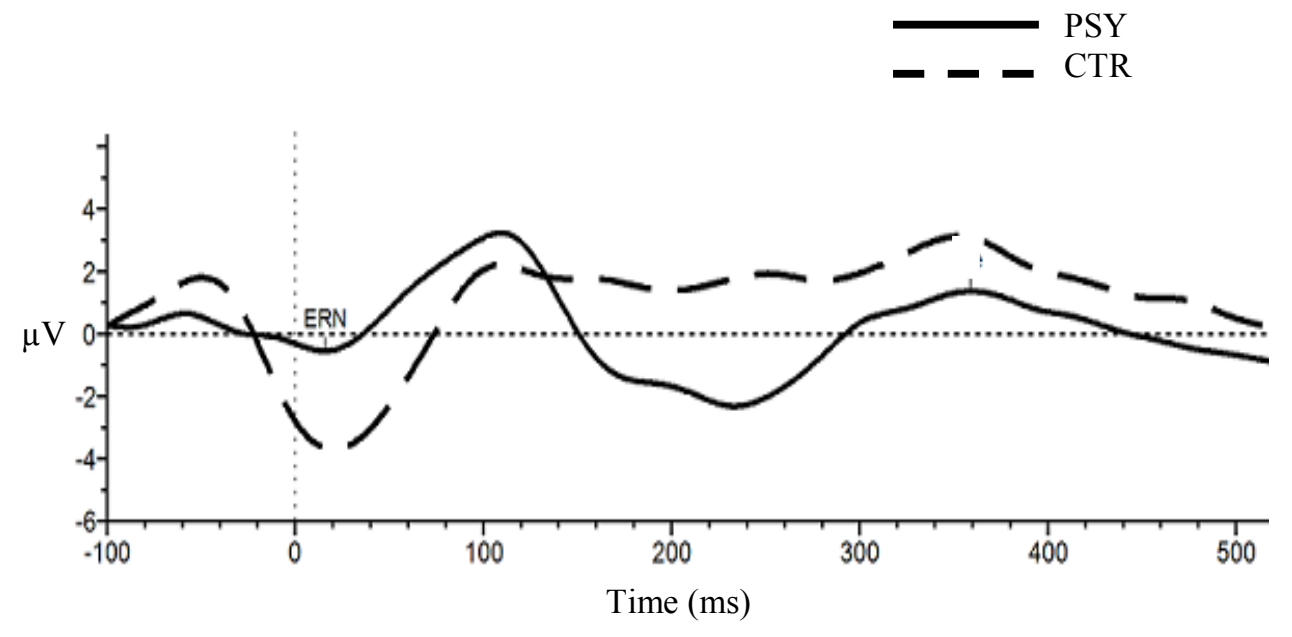

Figure 3: Grand average waveforms at the FCz site. PSY = individuals with a history of psychosis; $\mathrm{CTR}=$ comparison controls; $\mathrm{ERN}=$ error-related negativity; $\mathrm{Pe}=$ error positivity.

A planned comparison $t$-tests revealed that the PSY group had significantly reduced Pe amplitude during errors at $\mathrm{Cz}, t(25)=2.75, p=.013$, cohen's $d=1.12$, but not at the Pz site, $t(25)=.885, p=.384$, cohen's $d=.34$ or during correct responses at either the Cz site, $t(25)=$ $.231, p=.819$, cohen's $d=.09$ or the Pz site, $t(25)=.000, p=1.0$, cohen's $d=0$. A 2 (Group) X 2 (Accuracy of trials) X 2 (Site) mixed ANOVA for the Pe revealed a significant Accuracy by Site interaction, $F(1,25)=10.15, p=.004, \eta^{\prime} p^{2}=.289$. Post hoc analyses compared the Pe for correct and incorrect trials separately at each site across all participants. Results revealed that the Pe amplitude at $\mathrm{Pz}$ is significantly greater than the Pe amplitude at $\mathrm{Cz}, F(1,26)=7.57, p=.011$, $\eta^{\prime} p^{2}=.225$ for correct trials, but not for error trials $F(1,26)=.020, p=.888, \dot{\eta} p^{2}=.001$. The Group X Accuracy interaction approached significance, $F(1,25)=3.96, p=.058$. Mean 
amplitude of the Pe during error and correct trials are presented in Figure 4.

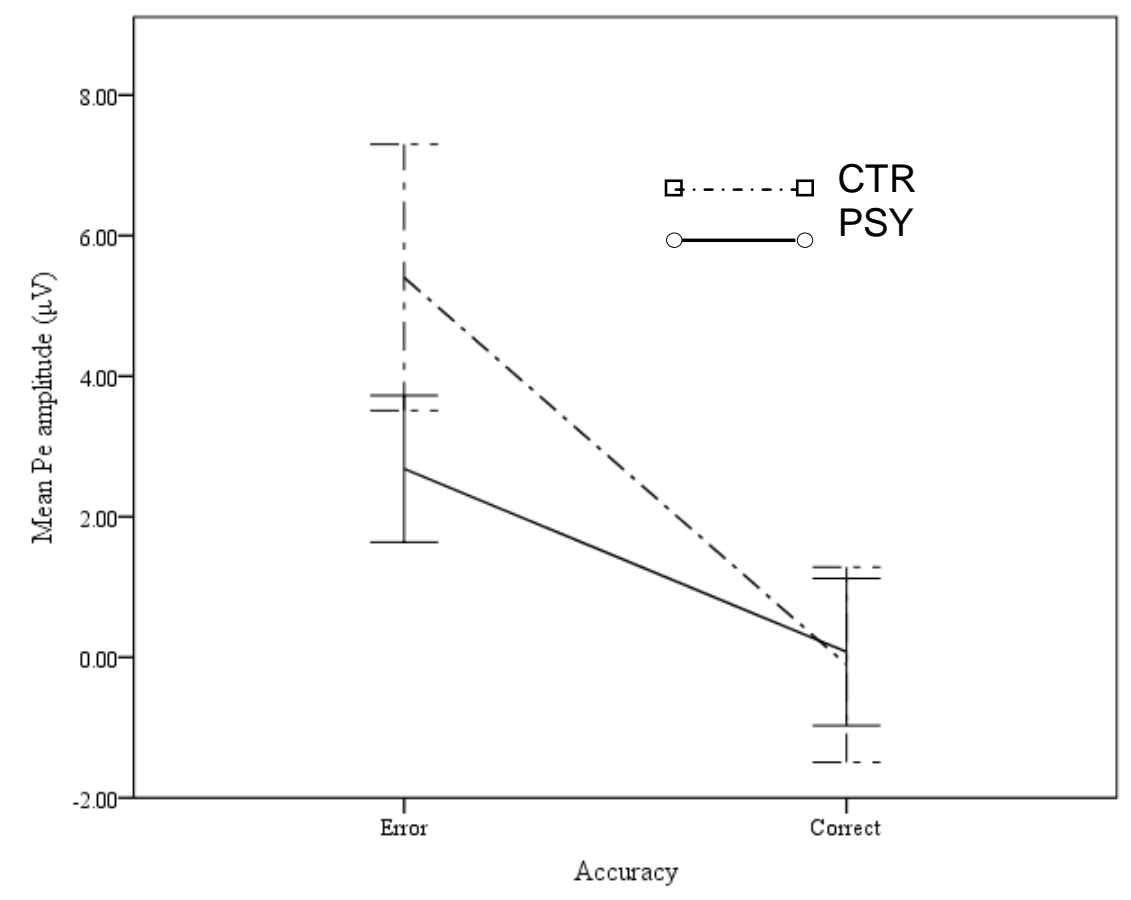

Figure 4: Mean amplitude of the Pe at site $\mathrm{Cz}$ during error and correct trials. PSY = individuals with a history of psychosis; CTR = comparison controls. Error bars represent $95 \%$ confidence interval.

\section{ERP Amplitude Correlation with Schizotypy}

ERN amplitude across the entire sample was correlated with the three factors of the SPQ$\mathrm{BR}$ as it is a continuous measure of traits across a spectrum ranging from normality to more severe psychopathology. ERN amplitude was associated with the Disorganized factor of the SPQ-BR at Fz, $r(24)=.50, p=.009$ (see Figure 5) and FCz, $r(25)=.48, p=.011$, as well as with the total SPQ-BR Total score at Fz, $r(24)=.41, p=.036$ and FCz, $r(25)=.39, p=.048$. Because the ERN amplitude is measured as a negative value, the positive correlation coefficients indicate an inverse relationship (i.e., the more reduced the ERN amplitude, the greater the SPQ score). 
The ERN was not significantly associated with the Cognitive Perceptual or the Interpersonal factors of the SPQ-BR. Furthermore, the Pe amplitude for error trials at the $\mathrm{Cz}$ site was inversely correlated with the Cognitive Perceptual factor of the SPQ-BR, $r(25)=-.383, p=.049$ (see Figure 6). The CRN at either site and the Pe at Pz were not associated with any factors of the SPQ-BR (all $p>.05)$.

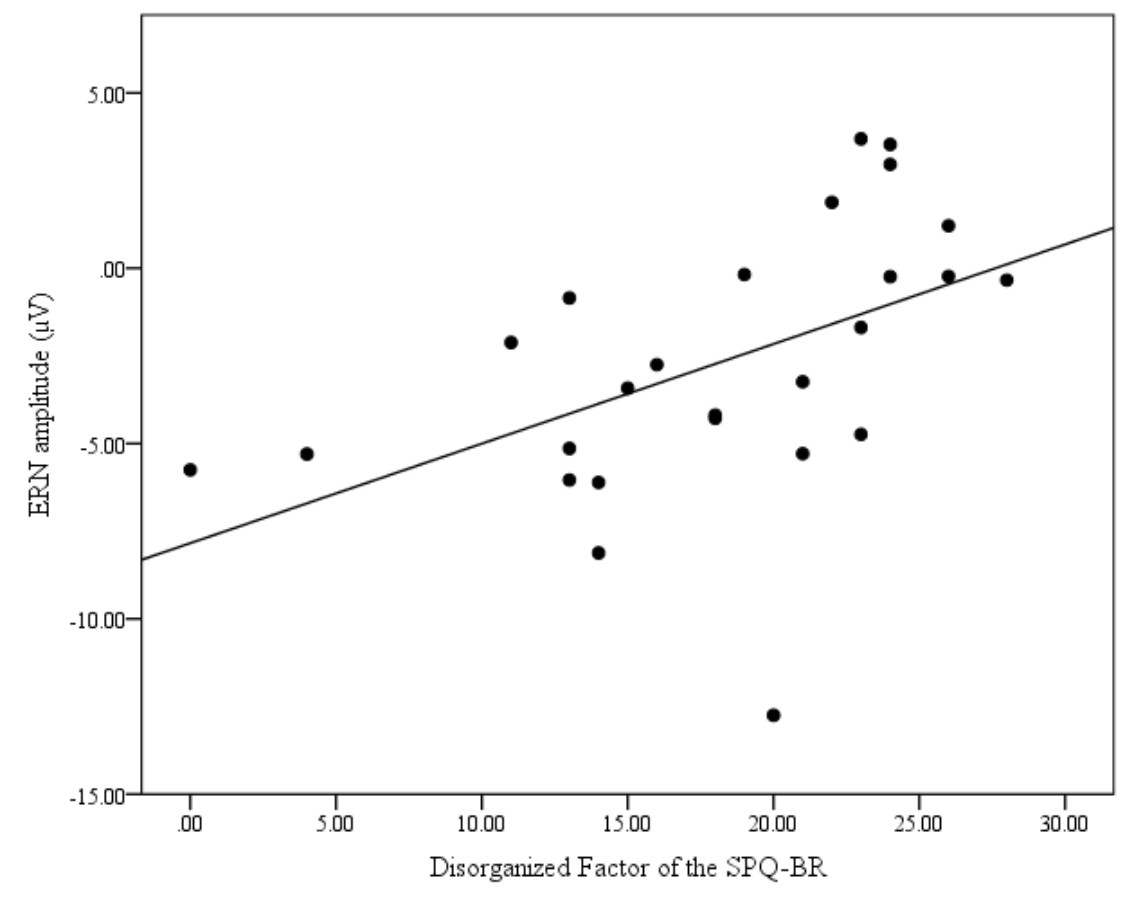

Figure 5: Correlation between the SPQ-BR Disorganized factor and ERN amplitude. Data displayed are from the Fz site. The positive correlation coefficient indicate an inverse relationship due to the ERN being measured as a negative value. 


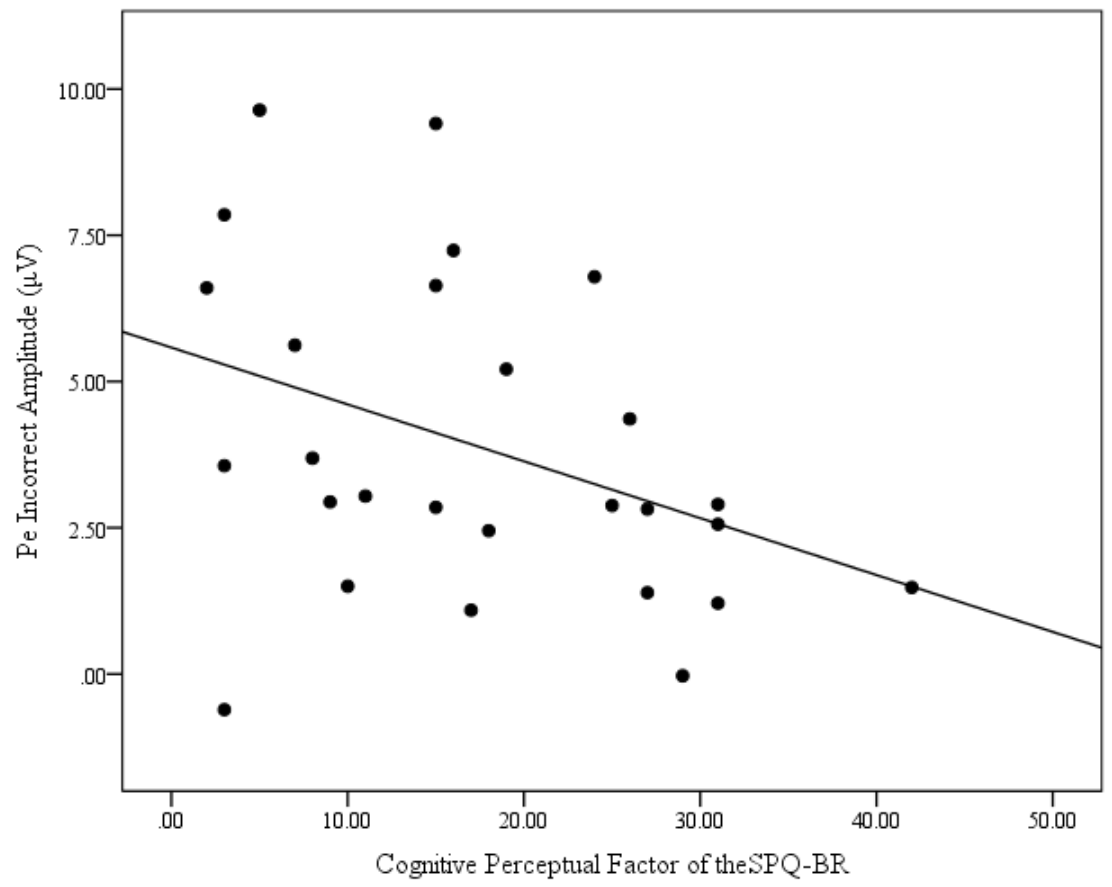

Figure 6: Correlation between the SPQ-BR Cognitive Perceptual Factor and Pe amplitude. Data displayed are from the $\mathrm{Cz}$ site. 


\section{CHAPTER 4: DISCUSSION}

In support of our hypothesis and existing results, individuals with a history of psychosis exhibited reduced ERN amplitude. This finding was more prominent at the FCz site than the Fz site, which suggests that FCz may be a superior site for distinguishing between individuals with a history of psychosis and those without. Contrary to our hypothesis, we failed to find differences in CRN amplitude at either site. Unlike the regularity of finding reduced ERN in schizophrenia, CRN findings in the literature have been mixed, with studies finding increased, decreased, or no difference in CRN amplitudes. It has been suggested that differences in CRN may be related to task difficulty (Mathalon et al., 2009). Although a difference in CRN amplitude has been reported between schizophrenia and nonpsychiatric controls during the flanker task in another study (Morris, Yee, \& Nuechterlein, 2006), it was found during the speeded condition, in which the accuracy rates were between $39-82 \%$, but not during the accuracy condition, in which the accuracy rates were generally greater than $90 \%$. The mean accuracy rate in the present study was approximately $91 \%$, suggesting that the task was relatively easy for our participants and may have produced less partial errors.

Also contrary to our hypothesis and existing literature, the Pe amplitude at site $\mathrm{Cz}$ was significantly blunted for error trials in the PSY group, but not for correct trials. Most studies have found no difference in Pe amplitude for neither error nor correct trials between controls and patients with schizophrenia, and one study found reduced Pe amplitude as calculated by a difference wave (error minus correct response) in schizophrenia, but not in those with other psychotic disorders (Foti et al., 2013). One possibility for this difference is that $60 \%$ of the individuals in the PSY group were diagnosed with either schizophrenia or schizoaffective 
disorder, and may have driven the difference similar to the study by Foti and colleagues. Another possibility for the difference is within the control group, which included individuals with a number of diagnoses. Pe activity may be different in the comparison controls in this study when compared with other studies where controls were without any diagnosis. This has been shown in the ERN, where individuals with anxiety disorders have demonstrated enhanced activity (Hajcak \& Simons, 2002; Weinberg, Klein, \& Hajcak, 2012).

ERN amplitude reduction was associated with a higher score on the Disorganized factor of the SPQ-BR but not the Cognitive Perceptual or the Interpersonal factor. This finding supports the idea that error monitoring deficits may lead to the inability to organize thoughts (McGrath, 1991). The association between reduced ERN amplitude and conceptual disorganization as measured by the Brief Psychiatric Rating Scale has been found previously trending significance in individuals with schizophrenia (Mathalon et al., 2002). They also found smaller ERN and CRN amplitudes were related to more severe hallucinatory behavior. Foti et al. (2013) found an inverse relationship between ERN amplitude and negative symptoms of the Scale for the Assessment of Negative Symptoms, but not psychotic or disorganized symptoms in a patient group consisting of individuals with schizophrenia and other psychotic disorders. We also found that reduced $\mathrm{Pe}$ amplitude at $\mathrm{Cz}$ was related to a higher score on the Cognitive-Perceptual factor of the SPQ-BR. The pattern of abnormality presently found in the ERP amplitudes was reflected in SPQ-BR scores, that is, ERN and Pe amplitude reductions found in PSY and were also associated with greater SPQ-BR scores, whereas CRN amplitude appeared normal and was also unrelated to SPQ-BR scores. ERN and Pe may be ERP components specifically related to psychosis. Nonetheless, further work is necessary to clarify associations between error-related 
ERP activities and clinical symptoms and personality traits.

Consistent with the exiting literature and in support of our hypothesis, individuals with a history of psychosis were less accurate in identifying their errors, although they did not exhibit difficulties identifying their correct responses. Overconfidence in errors have been found in schizophrenia across various cognitive tasks, including memory (Moritz \& Woodward, 2006b; Moritz, Woodward, \& Chen, 2006), decision making (Moritz et al., 2009), and social cognition (Köther et al., 2012). Supporting evidence from the current response inhibition task suggests a general error monitoring deficit. It is noteworthy that all of the significant findings in the current study are associated with error responses, suggesting that individuals with a history of psychosis have a specific deficit in monitoring of their errors that is apparent at the subjective level and at the psychophysiological level. From a risk management perspective, identifying errors, and thus prompting reevaluation or a change in behavior, is more important than acknowledging correct behavior. The impact of this may be even more significant in individuals with severe mental illness, such as in the case of remembering to take the correct medications and going to a clinical appointment.

This study was limited by a small sample size. Although efforts were made to increase the difficulty of the task, data from many participants who completed the study were excluded due to not having enough errors. Some studies using the flanker task have used an individually customized reaction time window, which generated more errors. It is also difficult to compare the results of the current study with existing literature on error-related ERP amplitudes, as most studies included exclusively individuals with a diagnosis of schizophrenia. One study examined ERN in individuals with psychosis, those with schizophrenia, and healthy controls and found 
similar reduction in ERN in both patient groups, but a blunted Pe in schizophrenia compared with the other psychotic disorders group (Foti, et al., 2012). The present study focuses on error monitoring in psychosis across diagnostic categories, although other symptoms that are also associated with schizophrenia (e.g., negative symptoms, neurocognitive deficits) may also be associated with the error monitoring system.

Despite these limitations, the present study used a relatively novel transdiagnostic approach, which adds to the existing findings using schizophrenia samples, to show that abnormalities in error-related brain activity are not limited to individuals with schizophrenia, but are present in individuals with a history of psychosis across diagnoses. Error monitoring deficits may be a marker for psychosis proneness and may predict psychotic disorders. In addition, deficits in error monitoring may moderate the relationship between symptomology and functional outcome. These findings are relevant in individuals with psychosis regardless of diagnosis and in individuals at risk for developing psychosis, and have the potential to inform targets for psychosocial intervention, perhaps focusing on feedback to improve self-monitoring and identification of errors. Further work in this area is necessary to identify specific pathways through which error monitoring moderates the relationship between clinical symptoms and functional outcome. 
APPENDIX: IRB APPROVAL LETTER 
University of Central Florida Institutional Review Board Office of Research \& Commercialization

12201 Research Parkway, Suite 501

Orlando, Florida 32826-3246

Telephone: 407-823-2901 or 407-882-2276

www.research.ucf.edu/compliance/irb.html

\section{Approval of Human Research}

From: $\quad$ UCF Institutional Review Board \#1

FWA00000351, IRB00001138

To: Jeffrey S. Bedwell

Date: $\quad$ May 21, 2012

Dear Researcher:

On 5/21/2012, the IRB approved the following human participant research until 5/20/2013 inclusive:

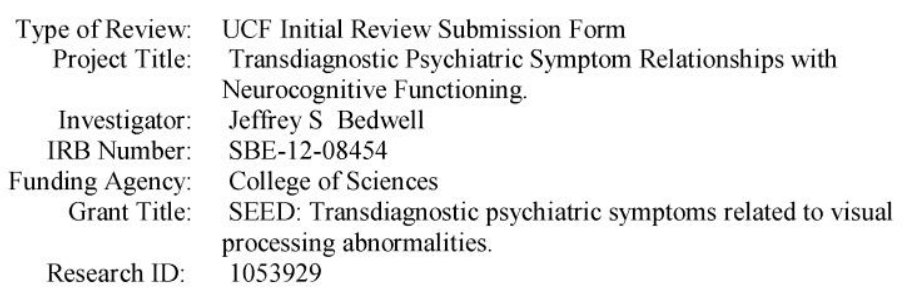

The Continuing Review Application must be submitted 30days prior to the expiration date for studies that were previously expedited, and 60 days prior to the expiration date for research that was previously reviewed at a convened meeting. Do not make changes to the study (i.e., protocol, methodology, consent form, personnel, site, etc.) before obtaining IRB approval. A Modification Form cannot be used to extend the approval period of a study. All forms may be completed and submitted online at https://iris.research.ucf.edu .

If continuing review approval is not granted before the expiration date of $5 / 20 / 2013$, approval of this research expires on that date. When you have completed your research, please submit a Study Closure request in iRIS so that IRB records will be accurate.

Use of the approved, stamped consent document(s) is required. The new form supersedes all previous versions, which are now invalid for further use. Only approved investigators (or other approved key study personnel) may solicit consent for research participation. Participants or their representatives must receive a copy of the consent form(s).

In the conduct of this research, you are responsible to follow the requirements of the Investigator Manual

On behalf of Sophia Dziegielewski, Ph.D., L.C.S.W., CF IRB Chair, this letter is signed by:

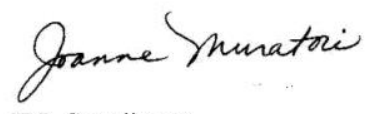

IRB Coordinator

Page 1 of 1 


\section{REFERENCES}

Alain, C., McNeely, H. E., He, Y., Christensen, B. K., \& West, R. (2002). Neurophysiological evidence of error-monitoring deficits in patients with schizophrenia. Cerebral Cortex, 12(8), 840-846.

Bates, A. T., Kiehl, K. A., Laurens, K. R., \& Liddle, P. F. (2002). Error-related negativity and correct response negativity in schizophrenia. Clinical Neurophysiology: Official Journal of the International Federation of Clinical Neurophysiology, 113(9), 1454-1463.

Bernstein, P. S., Scheffers, M. K., \& Coles, M. G. (1995). “Where did I go wrong?” A psychophysiological analysis of error detection. Journal of Experimental Psychology: Human Perception and Performance, 21(6), 1312-1322.

Carter, C. S., Braver, T. S., Barch, D. M., Botvinick, M. M., Noll, D., \& Cohen, J. D. (1998). Anterior cingulate cortex, error detection, and the online monitoring of performance. Science, 280(5364), 747-749.

Cohen, A. S., Matthews, R. A, Najolia, G. M., \& Brown, L. A. (2010). Toward a more psychometrically sound brief measure of schizotypal traits: introducing the SPQ-Brief Revised. Journal of Personality Disorders, 24(4), 516-537.

Coles, M. G., Scheffers, M. K., \& Holroyd, C. B. (2001). Why is there an ERN/Ne on correct trials? Response representations, stimulus-related components, and the theory of errorprocessing. Biological Psychology, 56(3), 173-189.

Debener, S., Ullsperger, M., Siegel, M., Fiehler, K., von Cramon, D. Y., \& Engel, A. K. (2005). Trial-by-trial coupling of concurrent electroencephalogram and functional magnetic resonance imaging identifies the dynamics of performance monitoring. The Journal of Neuroscience, 25(50), 11730-11737.

Dehaene, S., Posner, M. I., \& Tucker, D. M. (1994). Localization of a neural system for error detection and compensation. Psychological Science, 5(5), 303-305.

Eriksen, B., \& Eriksen, C. (1974). Effects of noise letters upon the identification of a target letter in a nonsearch task. Perception and Psychophysics, 16, 143-149.

Falkenstein, M., Hohnsbein, J., Hoormann, J., \& Blanke, L. (1991). Effects of crossmodal divided attention on late ERP components. II. Error processing in choice reaction tasks. Electroencephalography and Clinical Neurophysiology, 78(6), 447-455.

Falkenstein, M., Hoormann, J., Christ, S., \& Hohnsbein, J. (2000). ERP components on reaction errors and their functional significance: a tutorial. Biological Psychology, 51(2-3), 87-107. 
Foti, D., Kotov, R., Bromet, E., \& Hajcak, G. (2012). Beyond the broken error-related negativity: functional and diagnostic correlates of error processing in psychosis. Biological Psychiatry, 71(10), 864-872.

Foti, D., Kotov, R., \& Hajcak, G. (2013). Psychometric considerations in using error-related brain activity as a biomarker in psychotic disorders. Journal of Abnormal Psychology, 122(2), 520-531.

Frith, C. D. (1987). The positive and negative symptoms of schizophrenia reflect impairments in the perception and initiation of action. Psychological Medicine, 17(3), 631-648.

Frith, C. D., \& Done, D. J. (1989). Experiences of alien control in schizophrenia reflect a disorder in the central monitoring of action. Psychological Medicine, 19(2), 359-363.

Gawęda, L., Woodward, T. S., Moritz, S., \& Kokoszka, A. (2013). Impaired action selfmonitoring in schizophrenia patients with auditory hallucinations. Schizophrenia Research, 144(1-3), 72-79.

Gehring, W. J., Goss, B., Coles, M. G. H., Meyer, D. E., \& Donchin, E. (1993). A neural system for error detection and compensation. Psychological Science, 4(6), 385-390.

Green, M. F., Kern, R. S., Braff, D. L., \& Mintz, J. (2000). Neurocognitive deficits and functional outcome in schizophrenia: are we measuring the "right stuff"? Schizophrenia Bulletin, 26(1), 119-136.

Hajcak, G., McDonald, N., \& Simons, R. F. (2003). To err is autonomic: error-related brain potentials, ANS activity, and post-error compensatory behavior. Psychophysiology, 40(6), 895-903.

Hajcak, G., \& Simons, R. F. (2002). Error-related brain activity in obsessive - compulsive undergraduates. Psychiatry Research, 110, 63-72.

Holroyd, C. B., Nieuwenhuis, S., Yeung, N., Nystrom, L., Mars, R. B., Coles, M. G. H., \& Cohen, J. D. (2004). Dorsal anterior cingulate cortex shows fMRI response to internal and external error signals. Nature Neuroscience, 7(5), 497-498.

Hommes, J., Krabbendam, L., Versmissen, D., Kircher, T., van Os, J., \& van Winkel, R. (2011). Self-monitoring as a familial vulnerability marker for psychosis: an analysis of patients, unaffected siblings and healthy controls. Psychological Medicine, 1-11.

Horan, W. P., Foti, D., Hajcak, G., Wynn, J. K., \& Green, M. F. (2011). Impaired neural response to internal but not external feedback in schizophrenia. Psychological Medicine,42(8), 1637-1647. 
Kerns, J. G., Cohen, J. D., MacDonald, A. W., Johnson, M. K., Stenger, V. A., Aizenstein, H., \& Carter, C. S. (2005). Decreased conflict- and error-related activity in the anterior cingulate cortex in subjects with schizophrenia. The American Journal of Psychiatry, 162(10), 18331839.

Kircher, T. T. J., Koch, K., Stottmeister, F., \& Durst, V. (2007). Metacognition and reflexivity in patients with schizophrenia. Psychopathology, 40(4), 254-60.

Kopp, B., \& Rist, F. (1999). An event-related brain potential substrate of disturbed response monitoring in paranoid schizophrenic patients. Journal of Abnormal Psychology, 108(2), 337-346.

Koren, D., Seidman, L. J., Goldsmith, M., \& Harvey, P. D. (2006). Real-world cognitive--and metacognitive--dysfunction in schizophrenia: a new approach for measuring (and remediating) more "right stuff". Schizophrenia Bulletin, 32(2), 310-326.

Köther, U., Veckenstedt, R., Vitzthum, F., Roesch-Ely, D., Pfueller, U., Scheu, F., \& Moritz, S. (2012). "Don't give me that look" - overconfidence in false mental state perception in schizophrenia. Psychiatry Research, 196(1), 1-8.

Lysaker, P. H, Gumley, A., Luedtke, B., Buck, K. D., Ringer, J. M., Olesek, K., Kukla, M., et al. (2013). Social cognition and metacognition in schizophrenia: evidence of their independence and linkage with outcomes. Acta Psychiatrica Scandinavica, 127(3), 239247.

Lysaker, P. H, Shea, A. M., Buck, K. D., Dimaggio, G., Nicolò, G., Procacci, M., Salvatore, G., et al. (2010). Metacognition as a mediator of the effects of impairments in neurocognition on social function in schizophrenia spectrum disorders. Acta Psychiatrica Scandinavica, 122(5), 405-413.

Mathalon, D. H., Fedor, M., Faustman, W. O., Gray, M., Askari, N., \& Ford, J. M. (2002). Response-monitoring dysfunction in schizophrenia: An event-related brain potential study. Journal of Abnormal Psychology, 111(1), 22-41.

Mathalon, D. H., Jorgensen, K. W., Roach, B. J., \& Ford, J. M. (2009). Error detection failures in schizophrenia: ERPs and FMRI. International Journal of Psychophysiology, 73(2), 109117.

McGrath, J. (1991). Ordering thoughts on thought disorder. The British Journal of Psychiatry, 158, 307-316.

Metcalfe, J., \& Shimamura, A. P. (1994). Metacognition: Knowing About Knowing (p. 334). Cambridge: MIT Press. 
Miltner, W. H. R., Lemke, U., Weiss, T., Holroyd, C., Scheffers, M. K., \& Coles, M. G. H. (2003). Implementation of error-processing in the human anterior cingulate cortex: a source analysis of the magnetic equivalent of the error-related negativity. Biological Psychology, 64(1-2), 157-166.

Moritz, S, Veckenstedt, R., Randjbar, S., Hottenrott, B., Woodward, T. S., von Eckstaedt, F. V., Schmidt, C., et al. (2009). Decision making under uncertainty and mood induction: further evidence for liberal acceptance in schizophrenia. Psychological Medicine, 39(11), 18211829.

Moritz, S., Woodward, T. S., \& Chen, E. (2006). Investigation of metamemory dysfunctions in first-episode schizophrenia. Schizophrenia Research, 81(2-3), 247-252.

Moritz, S., Woodward, T. S., \& Ruff, C. C. (2002). Source monitoring and memory confidence in schizophrenia. Psychological Medicine, 33(1), 131-139.

Moritz, S., \& Woodward, T. S. (2006a). The contribution of metamemory deficits to schizophrenia. Journal of Abnormal Psychology, 115(1), 15-25.

Moritz, S., \& Woodward, T. S. (2006b). Metacognitive control over false memories: a key determinant of delusional thinking. Current Psychiatry Reports, 8(3), 184-190.

Moritz, S., Woodward, T. S., \& Chen, E. (2006). Investigation of metamemory dysfunctions in first-episode schizophrenia. Schizophrenia Research, 81(2-3), 247-252.

Morris, S. E., Yee, C. M., \& Nuechterlein, K. H. (2006). Electrophysiological analysis of error monitoring in schizophrenia. Journal of Abnormal Psychology, 115(2), 239-250.

Nelson, T., \& Narens, L. (1990). Metamemory: a theoretical framework and new findings. The Psychology of Learning and Motivation, 26, 125-173.

Nieuwenhuis, S., Ridderinkhof, K. R., Blom, J., Band, G. P., \& Kok, a. (2001). Error-related brain potentials are differentially related to awareness of response errors: evidence from an antisaccade task. Psychophysiology, 38(5), 752-760.

Penn, D. L., Corrigan, P. W., Bentall, R. P., Racenstein, J. M., \& Newman, L. (1997). Social cognition in schizophrenia. Psychological Bulletin, 121(1), 114-132.

Raine, A. (1991). The SPQ: a scale for the assessment of schizotypal personality based on DSMIII-R criteria. Schizophrenia Bulletin, 17(4), 555-564.

Scheffers, M. K., \& Coles, M. G. H. (2000). Performance monitoring in a confusing world: Error-related brain activity, judgments of response accuracy, and types of errors. Journal of Experimental Psychology: Human Perception and Performance, 26(1), 141-151. 
Silver, H., \& Goodman, C. (2007). Impairment in error monitoring predicts poor executive function in schizophrenia patients. Schizophrenia Research, 94(1-3), 156-163.

Silver, H., Goodman, C., Bilker, W., Gur, R. C., Isakov, V., Knoll, G., \& Feldman, P. (2006). Impaired error monitoring contributes to face recognition deficit in schizophrenia patients. Schizophrenia Research, 85(1-3), 151-161.

Vidal, F., Hasbroucq, T., Grapperon, J., \& Bonnet, M. (2000). Is the "error negativity" specific to errors? Biological Psychology, 51(2-3), 109-128.

Weinberg, A., Klein, D. N., \& Hajcak, G. (2013). Increased error-related brain activity distinguishes generalized anxiety disorder with and without comorbid major depressive disorder. Journal of Abnormal Psychology, 121(4), 885-896.

van Veen, V., \& Carter, C. S. (2006). Error detection, correction, and prevention in the brain: a brief review of data and theories. Clinical EEG and Neuroscience, 37(4), 330-335. 